\title{
Effect of the coadministration of citalopram with mirtazapine or atipamezole on rat contextual conditioned fear
}

This article was published in the following Dove Press journal:

Neuropsychiatric Disease and Treatment

II February 2014

Number of times this article has been viewed

\author{
Takahiro Masuda ${ }^{1,2}$ \\ Takeshi Inoue' \\ Yan An' \\ Naoki Takamura ${ }^{1,3}$ \\ Shin Nakagawa' \\ Yuji Kitaichi' \\ Tsukasa Koyama' \\ Ichiro Kusumi'
}

'Department of Psychiatry, Hokkaido University Graduate School of Medicine, Sapporo Japan; ${ }^{2}$ Medical Affairs, Dainippon Sumitomo Pharma, Co, Ltd, Tokyo, Japan; ${ }^{3}$ Regenerative and Cellular Medicine Office,

Dainippon Sumitomo Pharma, Co, Ltd, Osaka, Japan
Correspondence: Takeshi Inoue

Department of Psychiatry, Hokkaido University Graduate School of Medicine, North 15, West 7, Sapporo 060-8638, Japan

Tel $+8 I I I 7065160$

Fax +8I II706508I

Email tinoue@med.hokudai.ac.jp
Background: Mirtazapine, a noradrenergic and specific serotonergic antidepressant, which blocks the $\alpha_{2}$-adrenergic autoreceptors and heteroreceptors, has shown anxiolytic properties in clinical trials and preclinical animal experiments. The addition of mirtazapine to selective serotonin reuptake inhibitors (SSRIs) is clinically suggested to be more effective for anxiety disorders. In this study, we examined the combined effects of mirtazapine and citalopram, an SSRI, on the freezing behavior of rats, which was induced by contextual conditioned fear as an index of anxiety or fear.

Methods: Male Sprague Dawley rats individually received footshocks in a shock chamber, and 24 hours later, they were given citalopram and/or mirtazapine injections. One hour after citalopram and 30 minutes after mirtazapine administration, freezing behavior was analyzed in the same shock chamber without shocks.

Results: Mirtazapine decreased freezing in a dose-dependent manner, which is consistent with a previous report; it also enhanced an anxiolytic-like effect at a high dose $(30 \mathrm{mg} / \mathrm{kg})$ of citalopram. Because mirtazapine blocks $\alpha_{2}$-adrenoreceptors, the combined effect of atipamezole, a selective $\alpha_{2}$ receptor antagonist, with citalopram was also examined. Similar to mirtazapine, atipamezole reduced freezing dose-dependently, but the enhancement of citalopram's effects by atipamezole was not clear when compared with mirtazapine.

Conclusion: The present findings suggest that mirtazapine has an anxiolytic-like effect and may enhance the anxiolytic-like effect of SSRIs, but this enhancement may not be explained by its anti- $\alpha_{2}$ property alone.

Keywords: anxiety, conditioned fear, selective serotonin reuptake inhibitor, mirtazapine, $\alpha_{2}$-adrenoreceptor

\section{Introduction}

Selective serotonin reuptake inhibitors (SSRIs) are recommended as a first-line treatment by the recent treatment guidelines for anxiety disorders; benzodiazepines are the second-line treatment due to the patients' risks of developing drug dependency. ${ }^{1,2}$ However, not all patients with anxiety disorders remit even after adequate treatment; for instance, the remission rate was $45 \%$ in a clinical trial of one treatment in panic disorders. ${ }^{3}$ Such treatment-resistant anxiety disorders have not been studied enough. ${ }^{2}$ The "switching to" or "augmenting by" drugs with different pharmacological profiles are recommended as the third or fourth treatment by the treatment guideline. Among them, mirtazapine, a noradrenergic and specific serotonergic antidepressant that blocks the $\alpha_{2}$-adrenergic autoreceptors, heteroreceptors, and serotonin $(5-\mathrm{HT})_{2 \mathrm{C} / 2 \mathrm{~A} / 3}$ receptors, ${ }^{4}$ is a third or fourth line of treatment option for panic disorder, obsessive-compulsive 
disorder, and posttraumatic stress disorder, as indicated by the recent treatment guidelines. ${ }^{2}$

Because mirtazapine is often chosen as an augmentation therapy of SSRIs for major depression, ${ }^{5-7}$ mirtazapine augmentation of SSRIs also seems promising for treatmentresistant anxiety disorders, but only one single-blind study reported that adding mirtazapine to citalopram resulted in a faster onset of efficacy for obsessive-compulsive disorder. ${ }^{8}$ As mentioned above, because the pharmacological profile of mirtazapine is quite different from those of SSRIs, ${ }^{4}$ the addition of mirtazapine may augment the anxiolytic effect of SSRIs. A recent preclinical study using in vivo microdialysis supported this idea; this study showed that mirtazapine itself increased 5-HT neurotransmission in the hippocampus and enhances 5-HT neurotransmission increased by the serotonin noradrenaline reuptake inhibitor (SNRI) milnacipran in the hippocampus and prefrontal cortex. ${ }^{9}$ Increased 5-HT neurotransmission is related to the anxiolytic effect of antidepressants, including SSRIs ${ }^{10,11}$ and possibly mirtazapine. ${ }^{9}$

Preclinical studies using animal models of anxiety are necessary before the clinical introduction of new pharmacological treatments or their combinations are used to see whether their putative efficacy in animals can be confirmed, and to determine whether the mechanism of action can be clarified. However, efficacy studies of the putative anxiolytic effect of mirtazapine in animal anxiety models have rarely been performed in contrast to preclinical studies of SSRIs. ${ }^{11}$ Only one study by Kakui et $\mathrm{al}^{12}$ reported that mirtazapine has an anxiolytic-like effect in the rat contextual conditioned fear model, and this effect is mediated by activation of the $5-\mathrm{HT}_{1 \mathrm{~A}}$ receptor and the $\alpha_{1}$-adrenoreceptor. Hence, a behavioral study to examine the anxiolytic-like effect of adding mirtazapine to SSRIs in animals has never been performed.

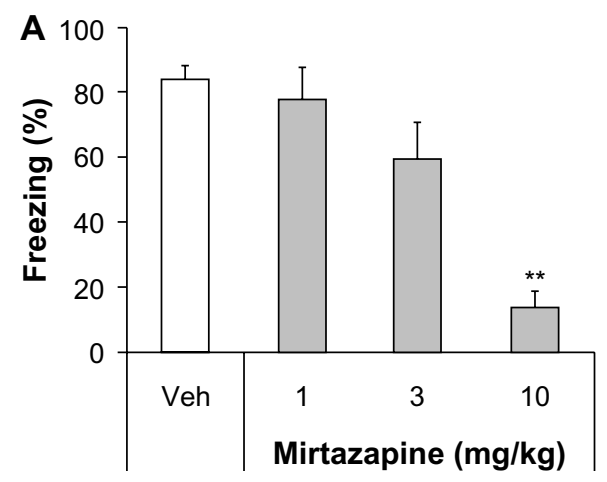

The aim of this study was to examine whether the combination of mirtazapine and the very specific SSRI, citalopram, ${ }^{4}$ increases the anxiolytic-like effect of citalopram on the expression of rat contextual conditioned fear. Specifically, drugs were administered to rats 24 hours after conditioning by footshock and shortly before the observation of freezing behavior. Furthermore, to clarify the role of the $\alpha_{2}$-adrenoreceptor in this effect, the effect of the highly selective $\alpha_{2}$-adrenoreceptor antagonist, atipamezole, ${ }^{13}$ was tested alone or in combination with citalopram.

\section{Methods}

\section{Animals}

Male Sprague Dawley rats (230-300 g) were obtained from Japan SLC, Inc. (Shizuoka, Japan). A total of 292 rats were used. The number of rats per group for each experiment is described in Figures 1-3. The rats were housed in polypropylene cages with wood shavings on the floor; there were four animals per cage, with free access to food and water. The subjects were maintained under a 12-hour light-dark cycle (light phase: 6.30 am to $6.30 \mathrm{pm}$ ) in a temperature-controlled environment $\left(22^{\circ} \mathrm{C} \pm 1^{\circ} \mathrm{C}\right)$. The rats were tested during the light phase. Experiments began after a 2-week period of acclimatization. All experiments were performed between $8.00 \mathrm{am}$ and $1.00 \mathrm{pm}$. All experiments were approved by the Hokkaido University School of Medicine Animal Care and Use Committee, and they were conducted in compliance with the Guide for the Care and Use of Laboratory Animals, Hokkaido University School of Medicine.

\section{Drugs}

Citalopram hydrobromide (a gift from Dainippon Sumitomo Pharma Co, Ltd, Osaka, Japan) was dissolved in

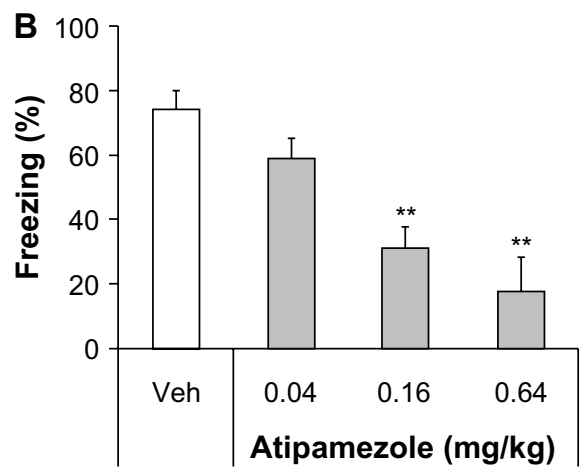

Figure I Effect of acute mirtazapine or atipamezole treatment on conditioned freezing in rats.

Notes: One day after footshock, mirtazapine $(1-10 \mathrm{mg} / \mathrm{kg})$ or atipamezole $(0.04-0.64 \mathrm{mg} / \mathrm{kg})$ was administrated 60 minutes before testing intraperitoneally or subcutaneously, respectively. Data are expressed as the mean \pm standard error of the mean of freezing scored for a 5 -minute observation period. $* * P<0.01$ versus $V$ eh $($ Steel test). (A) $N=8$ (rats in each group); (B) $\mathrm{N}=16$ (rats in each group), except for the atipamezole $0.64 \mathrm{mg} / \mathrm{kg}$ group ( $\mathrm{N}=8$ ).

Abbreviations: Veh, vehicle; N, number. 

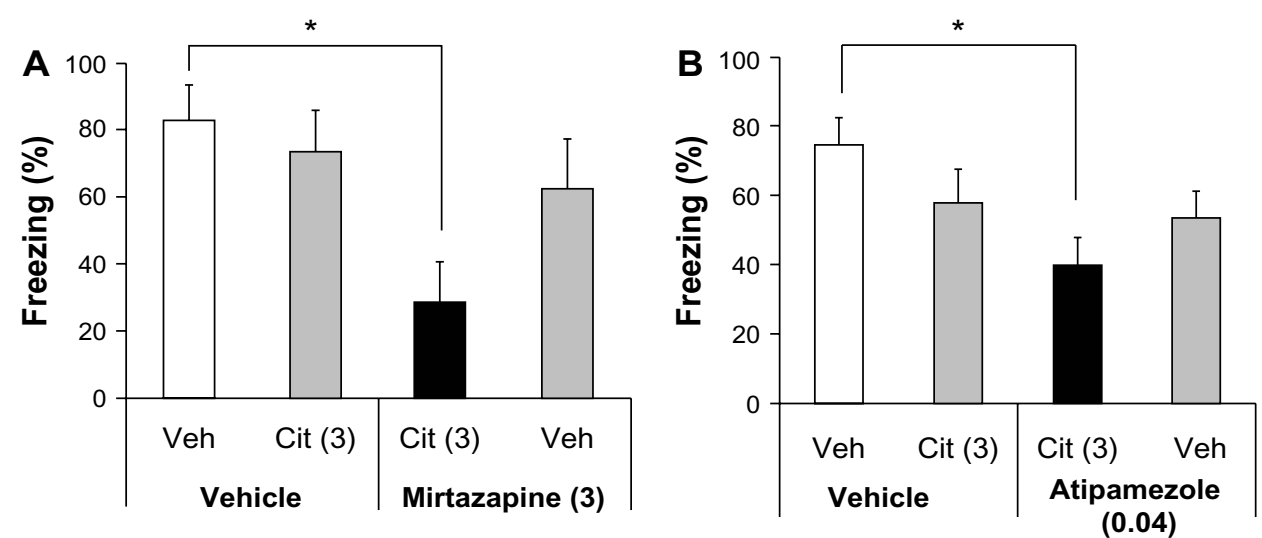

Figure 2 Effect of the coadministration of low-dose citalopram with subeffective doses of mirtazapine or atipamezole on conditioned freezing. Notes: One day after footshock, the low dose of citalopram ( $3 \mathrm{mg} / \mathrm{kg}$, subcutaneously) was administered I hour before testing. Mirtazapine ( $3 \mathrm{mg} / \mathrm{kg}$, intraperitoneally) or atipamezole $(0.04 \mathrm{mg} / \mathrm{kg}$, subcutaneously) was administrated 30 minutes after citalopram treatment (ie, 30 minutes prior to testing). Data are expressed as the mean \pm standard error of the mean of the freezing behavior scored during a 5 -minute observation period. $* \mathrm{P}<0.05$ versus $V$ eh $+\mathrm{Veh}$ (Steel-Dwass test). (A) $\mathrm{N}=7$ (rats in each group); (B) $\mathrm{N}=14$ (rats in each group).

Abbreviations: Veh, vehicle; Cit, citalopram; N, number.

$0.9 \%$ sterile saline. Atipamezole (Antisedan ${ }^{\circledR} 5 \mathrm{mg} / \mathrm{mL}$; Orion Pharma, Espoo, Finland) was diluted with distilled water. Mirtazapine (obtained from Merck and Co, Inc, Whitehouse Station, NJ, USA) was dissolved in $0.15 \%$ tartaric acid. The vehicle alone was administered as a control. Citalopram was administered 60 minutes before testing, and mirtazapine or atipamezole was administered 30 minutes before testing. Citalopram and atipamezole were injected subcutaneously at a volume of $1 \mathrm{~mL} / \mathrm{kg}$. Mirtazapine was injected intraperitoneally in a volume of $2 \mathrm{~mL} / \mathrm{kg}$. The doses and routes of administration of citalopram, mirtazapine, and atipamezole used in this study were determined based on our previous experiments ${ }^{11,12}$ and on earlier reports by other investigators..$^{14,15}$

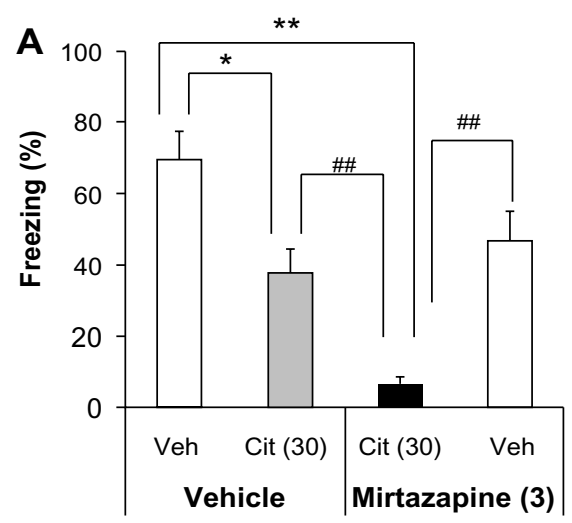

\section{Procedures of conditioned fear stress-induced freezing}

The total duration of the conditioning session was 5 minutes. The rats were individually subjected to inescapable electric footshocks for a total of 2.5 minutes in a shock chamber with a grid floor $(19 \times 22 \times 20 \mathrm{~cm}$; Medical Agent Co, Ltd, Tokyo, Japan). Five electric footshocks ( $2.5 \mathrm{~mA}$ unpredictable shock, each of a 30-second duration) were delivered at intershock intervals of 35-85 seconds (mean: 60 seconds) using a Model SGS-02D Shock Generator (Medical Agent Co, Ltd). One day after the footshocks, the rats were again placed in the shock chamber without footshocks and observed for 5 minutes. During the observation period, the duration of their freezing behavior was recorded using a modified

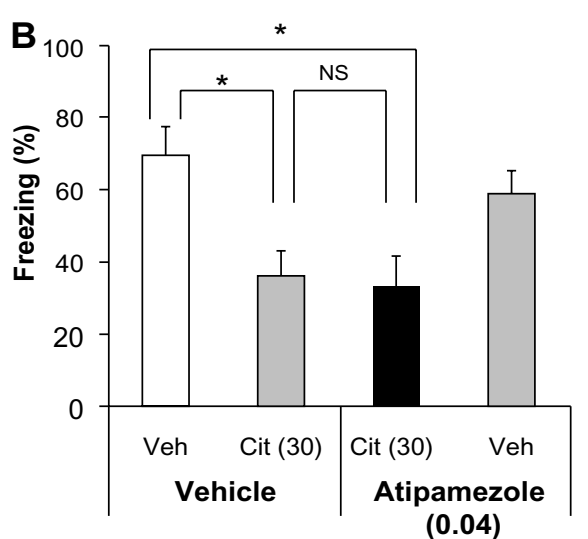

Figure 3 Effect of the coadministration of high-dose citalopram with subeffective mirtazapine or atipamezole on conditioned freezing.

Notes: One day after footshock, the high dose of citalopram $(30 \mathrm{mg} / \mathrm{kg}$, subcutaneously) was administered I hour before testing. Mirtazapine ( $3 \mathrm{mg} / \mathrm{kg}$, intraperitoneally) or atipamezole $(0.04 \mathrm{mg} / \mathrm{kg}$, subcutaneously) was administrated 30 minutes after citalopram treatment (ie, 30 minutes prior to testing). Data are expressed as the mean \pm standard error of the mean of freezing scored for a 5-minute observation period. ${ }^{*} P<0.05$; $* * P<0.0$ l, versus Veh + Veh; ${ }^{\# *} P<0.01$, versus Mirtazapine + Cit (Steel-Dwass test). (A) $\mathrm{N}=14$ (rats in each group); (B) $\mathrm{N}=16$ (rats in each group).

Abbreviations: Veh, vehicle; Cit, citalopram; N, number; NS, not significant. 
time-sampling procedure, as previously described. ${ }^{16}$ Every 10 seconds, the animal was classified as either freezing or active according to its behavior throughout the entire 10 -second period. Freezing was defined as the absence of all observable movement of the skeleton and the vibrissae, except those related to respiration. All other behaviors were scored as activity. The percentage scores for the duration of freezing behavior ( $\%$ freezing) were calculated for the 5-minute observation period.

In this study, six experiments, as shown in Figures 1-3, were performed independently. Each experiment was done once or twice, in which 28-32 rats, which included all groups, were tested on the same day to avoid the effect of variation between experiments. The rats were only used once for the experiments.

\section{Data analysis}

All the data are presented as the means \pm standard error of the mean of the individual values for each rat in all groups. The statistical analysis of the data was performed using the nonparametric Kruskal-Wallis test followed by the Steel test to compare each of the treatments with a single vehicle control in dose-response experiments, or with the Steel-Dwass test to compare all possible pairs of means in combined treatment experiments for multiple comparisons as a post hoc test.

\section{Results}

\section{Effect of acute mirtazapine}

or atipamezole treatment on the expression of conditioned freezing

Both mirtazapine and atipamezole affected the expression of conditioned freezing significantly (Kruskal-Wallis test, mirtazapine, $H=16.625, P<0.001$; atipamezole, $H=23.448$, $P<0.0001)$. A Steel post hoc test to compare each of the treatments with the vehicle control group revealed that mirtazapine showed significant inhibitory effects at $10 \mathrm{mg} / \mathrm{kg}(P<0.01$ versus the vehicle control group), but not at $1 \mathrm{mg} / \mathrm{kg}$ and $3 \mathrm{mg} / \mathrm{kg}$ (Figure 1A), and that atipamezole showed inhibitory effects at $0.16 \mathrm{mg} / \mathrm{kg}$ and at $0.64 \mathrm{mg} / \mathrm{kg}(P<0.01$ versus the vehicle control group), but not at $0.04 \mathrm{mg} / \mathrm{kg}$ (Figure 1B).

\section{Effect of coadministration of low- dose citalopram with mirtazapine or atipamezole on the expression of conditioned freezing}

We previously reported that acute treatment with the SSRI citalopram dose-dependently reduced the expression of conditioned freezing. ${ }^{17}$ At a low dose $(3 \mathrm{mg} / \mathrm{kg})$, citalopram did not change the expression of conditioned freezing significantly. ${ }^{17}$ On the other hand, at a high dose $(30 \mathrm{mg} / \mathrm{kg})$, citalopram showed the maximal inhibitory effect. ${ }^{17}$ In other words, even at a very high dose $(100 \mathrm{mg} / \mathrm{kg})$, the inhibitory effect of citalopram was equivalent to that of $30 \mathrm{mg} / \mathrm{kg} .{ }^{17}$ To investigate the combined effect of citalopram with mirtazapine or atipamezole, we used a subeffective low dose $(3 \mathrm{mg} / \mathrm{kg})$ of citalopram and subeffective doses of the other drugs (ie, $3 \mathrm{mg} / \mathrm{kg}$ of mirtazapine and $0.04 \mathrm{mg} / \mathrm{kg}$ of atipamezole). At each dose, any drug alone did not reduce freezing significantly. The coadministration of low-dose citalopram with subeffective doses of mirtazapine or atipamezole reduced the expression of conditioned freezing significantly (Kruskal-Wallis test, citalopram with mirtazapine, $H=8.808, P<0.05$; citalopram with atipamezole, $H=8.588$, $P<0.05$ ) (Figure 2). A Steel-Dwass post hoc test showed that coadministration of low-dose citalopram with subeffective doses of mirtazapine or atipamezole significantly reduced freezing compared with the controls (vehicle + vehicle versus citalopram + mirtazapine, $P<0.05$; vehicle + vehicle versus citalopram+ atipamezole, $P<0.05$ ).

\section{Effect of the coadministration of high- dose citalopram with mirtazapine or atipamezole on the expression of conditioned freezing}

To investigate whether the maximal inhibitory effect of citalopram is augmented by mirtazapine or atipamezole, we used a high dose $(30 \mathrm{mg} / \mathrm{kg})$ of citalopram with subeffective doses of mirtazapine $(3 \mathrm{mg} / \mathrm{kg})$ or atipamezole $(0.04 \mathrm{mg} / \mathrm{kg})$. Coadministration of high-dose citalopram with subeffective mirtazapine or atipamezole doses significantly reduced the expression of conditioned freezing (Kruskal-Wallis test, citalopram with mirtazapine, $H=27.223, P<0.0001$; citalopram with atipamezole, $H=13.971, P<0.005$ ) (Figure 3). A Steel-Dwass post hoc test showed that both high-dose citalopram alone and the coadministration of high-dose citalopram with subeffective mirtazapine significantly reduced freezing compared with the controls (vehicle + vehicle versus citalopram + vehicle, $P<0.05$; vehicle + vehicle versus citalopram + mirtazapine, $P<0.01$ ). Moreover, the coadministration of high-dose citalopram with subeffective mirtazapine significantly reduced freezing compared with high-dose citalopram alone and mirtazapine alone (citalopram + vehicle versus citalopram + mirtazapine, $P<0.01$; citalopram + mirtazapine versus vehicle + mirtazapine, $P<0.01$; Steel-Dwass 
post hoc test) (Figure 3A). Similarly, both high-dose citalopram alone and the coadministration of high-dose citalopram with subeffective doses of atipamezole also significantly reduced freezing compared with controls (vehicle + vehicle versus citalopram + vehicle, $P<0.05$; vehicle + vehicle versus citalopram + atipamezole, $P<0.05)$. However, there was no significant difference in freezing between high-dose citalopram alone and the coadministration of high-dose citalopram with subeffective atipamezole (citalopram + vehicle versus citalopram + atipamezole, $P>0.05$, Steel-Dwass post hoc test) (Figure 3B).

\section{Discussion}

Consistent with our previous studies, ${ }^{12,18}$ mirtazapine reduced the expression of conditioned freezing dose-dependently, and the SSRI citalopram inhibited conditioned freezing. In this study, the combination of subeffective doses of mirtazapine $(3 \mathrm{mg} / \mathrm{kg}$ ) and citalopram ( $3 \mathrm{mg} / \mathrm{kg}$ ) enhanced the inhibitory effect of both drugs on conditioned freezing. Our previous study showed that high-dose citalopram $(30 \mathrm{mg} / \mathrm{kg})$ produces the maximal effect on conditioned freezing. ${ }^{17}$ In this study, adjunctive subeffective mirtazapine enhanced the maximal inhibitory effect of high-dose citalopram $(30 \mathrm{mg} / \mathrm{kg})$ on conditioned freezing. These results suggest that mirtazapine itself has an anxiolytic-like effect, and that mirtazapine enhances the anxiolytic-like effect of SSRIs. This study is the first study to show that the combination of mirtazapine and an SSRI augments the anxiolytic-like effects of each drug. Based on these experimental findings, the putative efficacy of the combination therapy with mirtazapine and SSRI for treatment-resistant anxiety disorders may be promising, as this combination therapy is adapted to treatment-resistant forms of major depression. ${ }^{6}$

In contextual conditioned fear in rats, drugs that facilitate 5-HT neurotransmission (ie, that increase extracellular 5-HT levels in the brain) generally reduce conditioned freezing. ${ }^{11}$ Based on this principle, the previous findings of in vivo microdialysis studies may account for the augmentation effect of the combined mirtazapine and an SSRI. ${ }^{9,19}$ There are conflicting results as to whether mirtazapine alone increases extracellular 5-HT levels in the brain; some studies reported that mirtazapine increased extracellular 5-HT levels in the hippocampus, ${ }^{9,19}$ but others reported negative results in the hippocampus and other forebrain regions. ${ }^{20,21}$ Interestingly, mirtazapine enhances the increasing effect of the SNRI, milnacipran, on extracellular 5-HT levels in the hippocampus and prefrontal cortex. ${ }^{9}$ Hence, these previous findings suggest that the augmentation effect of the combined mirtazapine and SSRI in contextual conditioned freezing is mediated by more increases in extracellular 5-HT. Moreover, 5-HT $1 \mathrm{~A}$ receptors are a potentially strong candidate for the target receptors, in which the anxiolytic-like activities of mirtazapine alone and its combination with an SSRI is mediated by an increase in 5-HT activity. It should be noted that 5- $\mathrm{HT}_{1 \mathrm{~A}}$ agonists reportedly reduced the expression of contextual conditioned freezing. ${ }^{11}$ Furthermore, approximately one-half of the anxiolytic-like effect of mirtazapine in the expression of contextual conditioned freezing was antagonized by the selective $5-\mathrm{HT}_{1 \mathrm{~A}}$ receptor antagonist, WAY-100635. ${ }^{12}$

As indicated in the introduction, mirtazapine blocks not only $\alpha_{2}$-adrenergic autoreceptors and heteroreceptors, but also $5-\mathrm{HT}_{2 \mathrm{C} / 2 \mathrm{~A} / 3}$ receptors. ${ }^{4}$ This study showed that the very selective $\alpha_{2}$ receptor antagonist, atipamezole, dosedependently reduced conditioned freezing at low doses, as did mirtazapine. An earlier study reported that another $\alpha_{2}$ receptor antagonist, yohimbine, also reduced the expression of conditioned freezing. ${ }^{22}$ The addition of subeffective doses of atipamezole to citalopram increased the inhibitory effect of low-dose citalopram, which was similar to what was shown with mirtazapine. A pharmacodynamic mechanism may be involved in this enhancement because atipamezole reportedly enhanced the effect of an SSRI and SNRI on 5-HT. ${ }^{15}$ Furthermore, the $\alpha_{2}$ receptor antagonist idazoxan, like mirtazapine, reportedly enhances the increases in extracellular 5-HT levels by the SNRI milnacipran in the prefrontal cortex. ${ }^{9}$ However, the enhancement of citalopram's effects by atipamezole was not clear when compared with mirtazapine. Interestingly, atipamezole did not enhance the effect of high-dose citalopram, which induces the maximal effect, on conditioned freezing in contrast to mirtazapine. Therefore, although the effect of mirtazapine on conditioned freezing may be mediated by its $\alpha_{2}$ receptor antagonism, we cannot account for the enhancing effect of mirtazapine on citalopram only by the mechanism of $\alpha_{2}$ receptor antagonism.

Clinically, SSRIs and mirtazapine exert anxiolytic effects after chronic treatment; ${ }^{2}$ however, in the animal model, acute SSRI and mirtazapine exerted an anxiolyticlike effect in contextual conditioned fear. As we previously discussed in our review, ${ }^{11}$ this model is an animal model of fear or anxiety, but not of anxiety disorders. We found that prolonging the period between conditioning and exposure to conditioned fear stress for up to 7-14 days diminished the acute effects of SSRIs and mirtazapine, and the anxiolyticlike effects required chronic treatment (our published ${ }^{11}$ and unpublished data). Thus, the long interval between conditioning by footshock and exposure to conditioned 
fear stress might improve the predictive and face validities of contextual conditioned freezing as an animal model of anxiety disorders. In future studies, we plan to study the combined effect of mirtazapine and SSRIs on the expression of contextual conditioned freezing while using a longer interval between conditioning by footshock and exposure to conditioned fear stress.

\section{Conclusion}

This study shows that the anxiolytic-like effect of the SSRI citalopram in contextual conditioned fear is enhanced by the adjunctive subeffective dose of mirtazapine and, to a lesser extent, by the $\alpha_{2}$-adrenoceptor antagonist atipamezole. The combination of an SSRI and mirtazapine may be a promising strategy for treating anxiety disorders. In contextual conditioned fear, mirtazapine has an anxiolytic-like effect and may enhance the anxiolytic-like effect of SSRIs, but this enhancement may not be explained by its anti- $\alpha_{2}$ property alone.

\section{Acknowledgment}

This work was supported in part by a Grant-in-Aid for Scientific Research No 24591673 (Takeshi Inoue) from the Japanese Ministry of Education, Culture, Sports, Science and Technology (Tokyo, Japan).

\section{Disclosure}

Dr Takeshi Inoue has received honoraria from GlaxoSmithKline, Pfizer, Astellas, Eli Lilly, Mitsubishi Tanabe Pharma, Mochida Pharmaceutical, Otsuka Pharmaceutical, Meiji Seika Pharma, Asahi Kasei Pharma, Shionogi, Janssen Pharmaceutical, Takeda Pharmaceutical, and Yoshitomi Pharmaceutical; has received research/grant support from Otsuka Pharmaceutical; and is a member of the advisory boards of GlaxoSmithKline, Eli Lilly, Mochida Pharmaceutical, and Mitsubishi Tanabe Pharma. Dr Shin Nakagawa has received honoraria from GlaxoSmithKline, Eisai, Pfizer, DaiichiSankyo, Meiji Seika Pharma, Ono Pharmaceutical, and Eli Lilly, and has received research/grant support from Pfizer, Eli Lilly, Eisai, and Ono Pharmaceutical. Dr Ichiro Kusumi has received honoraria from Eli Lilly; has received research/ grant support from Takeda Pharmaceutical, Astellas, and Dainippon Sumitomo Pharma; and is a member of the advisory board of Dainippon Sumitomo Pharma and Tanabe Mitsubishi Pharma. Dr Tsukasa Koyama has received honoraria from GlaxoSmithKline, Astellas, and Eli Lilly; has received research/grant support from Astellas and GlaxoSmithKline; and is a member of the advisory boards of GlaxoSmithKline and Mitsubishi Tanabe Pharma. Dr Yuji Kitaichi has received honoraria from Otsuka Pharmaceutical and Meiji Seika Pharma. Dr Takahiro Masuda and Dr Naoki Takamura are employed by Dainippon Sumitomo Pharma. DrYan An reports no conflicts of interest in this work.

\section{References}

1. Irons J. Fluvoxamine in the treatment of anxiety disorders. Neuropsychiatr Dis Treat. 2005;1(4):289-299.

2. Bandelow B, Zohar J, Hollander E, et al; WFSBP Task Force on Treatment Guidelines for Anxiety, Obsessive-Compulsive and PostTraumatic Stress Disorders. World Federation of Societies of Biological Psychiatry (WFSBP) guidelines for the pharmacological treatment of anxiety, obsessive-compulsive and post-traumatic stress disorders - first revision. World J Biol Psychiatry. 2008;9(4):248-312.

3. Pollack MH, Lepola U, Koponen $\mathrm{H}$, et al. A double-blind study of the efficacy of venlafaxine extended-release, paroxetine, and placebo in the treatment of panic disorder. Depress Anxiety. 2007;24(1):1-14.

4. Millan MJ. Multi-target strategies for the improved treatment of depressive states: Conceptual foundations and neuronal substrates, drug discovery and therapeutic application. Pharmacol Ther. 2006;110(2): 135-170.

5. Furukawa TA, Akechi T, Shimodera S, et al. Strategic use of new generation antidepressants for depression: $\mathrm{SUN}\left({ }^{\wedge} \_\right) \mathrm{D}$ study protocol. Trials. 2011;12:116.

6. Papakostas GI. Managing partial response or nonresponse: switching, augmentation, and combination strategies for major depressive disorder. J Clin Psychiatry. 2009;70 Suppl 6:16-25.

7. Moret C. Combination/augmentation strategies for improving the treatment of depression. Neuropsychiatr Dis Treat. 2005;1(4):301-309.

8. Pallanti S, Quercioli L, Bruscoli M. Response acceleration with mirtazapine augmentation of citalopram in obsessive-compulsive disorder patients without comorbid depression: a pilot study. J Clin Psychiatry. 2004;65(10):1394-1399.

9. Yamauchi M, Imanishi T, Koyama T. A combination of mirtazapine and milnacipran augments the extracellular levels of monoamines in the rat brain. Neuropharmacology. 2012;62(7):2278-2287.

10. Erikkson E, Humble M. Serotonin in psychiatric pathophysiology. A review of data from experimental and clinical research. In: Pohl R, Gershon S, editors. Progress in Basic Clinical Pharmacology. Basel: Karger; 1990;3:66-119.

11. Inoue T, Kitaichi Y, Koyama T. SSRIs and conditioned fear. Prog Neuropsychopharmacol Biol Psychiatry. 2011;35(8):1810-1819.

12. Kakui N, Yokoyama F, Yamauchi M, et al. Anxiolytic-like profile of mirtazapine in rat conditioned fear stress model: Functional significance of 5-hydroxytryptamine 1A receptor and alpha1-adrenergic receptor. Pharmacol Biochem Behav. 2009;92(3):393-398.

13. Pertovaara A, Haapalinna A, Sirviö J, Virtanen R. Pharmacological properties, central nervous system effects, and potential therapeutic applications of atipamezole, a selective alpha2-adrenoceptor antagonist. CNS Drug Rev. 2005;11(3):273-288.

14. Haapalinna A, Viitamaa T, MacDonald E, et al. Evaluation of the effects of a specific alpha 2-adrenoceptor antagonist, atipamezole, on alpha 1and alpha 2-adrenoceptor subtype binding, brain neurochemistry and behaviour in comparison with yohimbine. Naunyn Schmiedebergs Arch Pharmacol. 1997;356(5):570-582.

15. Gobert A, Rivet JM, Cistarelli L, Melon C, Millan MJ. Alpha2adrenergic receptor blockade markedly potentiates duloxetine- and fluoxetine-induced increases in noradrenaline, dopamine, and serotonin levels in the frontal cortex of freely moving rats. J Neurochem. 1997;69(6):2616-2619.

16. Inoue $\mathrm{T}, \mathrm{Li} \mathrm{XB}, \mathrm{Abekawa} \mathrm{T}$, et al. Selective serotonin reuptake inhibitor reduces conditioned fear through its effect in the amygdala. Eur $J$ Pharmacol. 2004;497(3):311-316. 
17. Muraki I, Inoue T, Koyama T. Effect of co-administration of the selective 5-HT1A receptor antagonist WAY 100,635 and selective 5-HT1B/1D receptor antagonist GR 127,935 on anxiolytic effect of citalopram in conditioned fear stress in the rat. Eur J Pharmacol. 2008;586(1-3):171-178.

18. Hashimoto $\mathrm{S}$, Inoue $\mathrm{T}$, Koyama T. Serotonin reuptake inhibitors reduce conditioned fear stress-induced freezing behavior in rats. Psychopharmacology (Berl). 1996;123(2):182-186.

19. de Boer TH, Nefkens F, van Helvoirt A, van Delft AM. Differences in modulation of noradrenergic and serotonergic transmission by the alpha-2 adrenoceptor antagonists, mirtazapine, mianserin and idazoxan. J Pharmacol Exp Ther. 1996;277(2):852-860.
20. Bengtsson HJ, Kele J, Johansson J, Hjorth S. Interaction of the antidepressant mirtazapine with alpha2-adrenoceptors modulating the release of 5-HT in different rat brain regions in vivo. Naunyn Schmiedebergs Arch Pharmacol. 2000;362(4-5):406-412.

21. Millan MJ, Gobert A, Rivet JM, et al. Mirtazapine enhances frontocortical dopaminergic and corticolimbic adrenergic, but not serotonergic, transmission by blockade of alpha2-adrenergic and serotonin $2 \mathrm{C}$ receptors: a comparison with citalopram. Eur J Neurosci. 2000;12(3): 1079-1095.

22. Mueller D, Olivera-Figueroa LA, Pine DS, Quirk GJ. The effects of yohimbine and amphetamine on fear expression and extinction in rats. Psychopharmacology (Berl). 2009;204(4):599-606.

\section{Publish your work in this journal}

Neuropsychiatric Disease and Treatment is an international, peerreviewed journal of clinical therapeutics and pharmacology focusing on concise rapid reporting of clinical or pre-clinical studies on a range of neuropsychiatric and neurological disorders. This journa is indexed on PubMed Central, the 'PsycINFO' database and CAS.
The manuscript management system is completely online and includes a very quick and fair peer-review system, which is all easy to use. Visit http://www.dovepress.com/testimonials.php to read real quotes from published authors.

Submit your manuscript here: http://www.dovepress.com/neuropsychiatric-disease-and-treatment-journal 